\title{
XXIV.
}

\section{Kleinere Mitteilung.}

Nachtrag zu meinem Aufsatz über. Dysbasia angiosclerotica. (Diese Zeitschr. Bd. 32, Heft 2/3, S. 271).

Von

Dr. Idelsohn.

Nach Abfassung meines Aufsatzes - dessen Korrektur aus äusseren Gründen mir nicht übersandt worden ist - habe ich Gelegenheit gehabt, noch 8 weitere Fälle von D. a. zu beobachten; da mancher strittige Punkt durch sie teilweise geklärt werden kann, so möchte ich mir erlauben, dieselben hier kurz zu referieren. Es handelt sich um 8 kranke Männer ím Alter von $32,35,42,52,54,62,64$ und 66 Jahren, darunter 6 Juden, 2 Christen.

1. Bei dem jüngsten dieser Kranken, einem 32jährigen Juden, bestand Gangrän einzelner Zehen an beiden Füssen. Er hattê vor 10 Jahren Lues akquiriert, mangelhaft behandelt. Kein Abusus in Alcoholicis und Nikotin. Patient war noch im Frühjabr des vorigen Jahres von einem erfahrenen Neurologen untersucht worden, wobei nur eine Abschwächung des Pulses an einer Art. dorsal. pedis wahrscheinlich war, so dass gegen die "neurasthenischen Beschwerden" kalte Bäder verordnet wurden, die auch Patient gut absolvierte. Im darauf folgenden Winter Nágelbetteiterung an der 5. Zehe des rechten Fusses, Operation, im Anschluss daran im Frühjahr 1907 beginnende Gangrän; während des Krankenlagers Zyanose, heftige Schmerzen und beginnende Gangrän an den drei ersten Zehen links. Alle Pulse fehlen.

2. 42jähriger Mann, Jude. Vor 4 Jahren grosise Zehe des r. Fusses abgefroren, jetzt Gangrän. Am r. Fuss fehlen beide Pulse, an der 1. Hand fehlt der Radialpuls fast völlig. Ziemlich starker Raucher. Hat viel zu gehen.

3. 54jähriger Mann, Christ. Weder geraucht noch getrunken. Typische D. a. am l. Fuss mit fehlenden Pulsen; linker Radialpuls dentlich kleiner als rechter. Auch im rechten Fuss "Brennen" bei normalen Pulsen. Allgemeine Arteriosklerose: Hat viele Jahre im kalten Laden zu stehen gehabt.

4. 35 jähriger Mann, Jude. Vor 10 Jahren Lues, beginnende Tabes(?). Beiderseits fehlende Dorsalpulse. "Schwäche in den Beinen." Mässiger Raucher. Neurastheniker. Hat viel im Laden zu stehen. Nach spezifischer Behandlung: r. Dorsalpuls fadenförmig palpierbar, Funktion bedeutend besser. Miosis, Pupillenstarre, Gürtelgefühl, ein Achillesreflex fehlt, Patellarreflex nur mit Jendrassik auszulösen. Zugänghiche Arterien sklerosiert: 
5. 64jähriger Mann, Jude. Beiderseits Plattfuss. Der rechte Dorsalpuls unsicher zu fühlen, alle übrigen Fusspulse fehlen. Typische Beschwerden erst links gehabt, dann spontane Besserung seit 2 Jahren; jetzt rechts typische D. a., Angina pectoris, Tibialödem, Cor nach beiden Seiten dilatiert, Zucker 2,4 Proz. Rauchte mässig, trank fast gar nicht. Sitzt seit vielen Jahren in einem kalten Geschäftslokal.

6. 62jähriger Arzt, Jude. Konstitutioneller Neuropath. Hat früher "gut gelebt". Vor einem Jahr typische D. a. am r. Bein, seit einem halben Jahr keine Beschwerden. Allgemeine Arteriosklerose. Im Harn wechselnde Mengen von Albumen und Saccharum. Passagere aphasische und dysphasische Zustände, leichter Grad von Demenz. Am r. Fuss fehlen die Pulse.

7. 52jähriger Buchbindermeister, Christ. Mässiger Raucher. Hat viel zu stehen. Vor 2 Jahren typische D. a. am 1. Fuss. Die Pulse fehlen links. Spontane Besserung resp. Heilung. Patient kommt wegen allgemeiner Nervosität in die Sprechstunde. Leicht euphorisch. (Beginnende Gehirnsklerose?)

8. 66jähriger Mann, Christ. Als Nebenbefund: L. Dorsalpuls vorhanden, alle übrigen Fusspulse fehlen. Am r. Fuss Varizen, Phleholithen, eingewachsene Nägel und senile Hautveränderungen an beiden Füssen. Keinerlei Gehstörung besteht oder hat bestanden. Allgemeine Arteriosklerose.

Aus den angeführten Beobachtungen geht wiederum hervor, wie verschieden sich der Verlauf der D. a. bei alten und bei jugendlichen Individuen gestaltet. Im Fall 1 sehen wir bei einem jungen Individuum im Laufe eines Jahres die Pulse verschwinden und Gangrän eintreten, während in Fall 8 trotz des hohen Alters and der allgemeinen Arteriosklerose es nicht nur nicht zur Gangrän gekommen ist, sondern es fehlen sogar jegliche subjektive Beschwerden in den Füssen. Ebenso der schnelle und bösartige Verlauf in Fall 2 und die spontane Besserung in Fall 5, 6 und 7. Ich habe übrigens in einem Siechenhause (Bicêtre-Prof. Pierre Marie) gar nicht selten die Fusspulse bei Kranken vermisst, die wegen anderweitiger Erscheinungen in die Krankenstation aufgenommen worden waren und die über keinerlei Störungen der Gehfähigkeit zu klagen hatten. Es scheint sich demnach bei alten, an allgemeiner Arteriosklerose leidenden Kranken eine kompensatorische Blutdrucksteigerung herauszubilden, die den schlecht ernährten distalen Gewebsbezirken zugute kommt und das Auftreten der Gangrän verhindern kann. Das Fehlen der Fusspulse an sich ist ja noch kein Zeichen der unterbrochenen Zirkulation, dieselbe ist nur nicht so ausgiebig, dass die Arterienwand füblbar gehoben wird; doch wird die Ernährung durch diese Arterien dank dem erhöhten Blatdruck noch hinreichend unterhalten. Umgekehrt muss man annehmen, dass bei dem Fehlen der Fusspulse bei jugendlichen Individuen das verengte Lumen der Arterie bei dem nicht erhöhten allgemeinen Blutdruck die durchfliessende Blutmenge nicht in die distalsten Kapillargebiete eindringen lässt und dass auf diese Weise das Zustandekommen der Gangrän bei den jugendlichen Individuen - begünstigt wird.

Diese mechanischen Bedingungen dürften auch bei den spontanen Besserungen, die vorzugsweise ältere Kranke betrafen, im Spiele sein, 
ebenso könnten sie das Auftreten von Erscheinungen der D. a. bei alten Leuten verhindern.

Unter den ätiologischen Faktoren verdient die Kälte, auf die schon von anderen Autoren vielfach hingewiesen wurde, eine besondere Beachtung, doch scheint nicht der niedrige Kältegrad so sehr als der wiederholte Kältereiz (im Laden, dessen Tür beständig auf- und zugeht) das vasomotorische System zu schädigen. Ebenso schwerwiegend ist der Einfluss des anhaltenden Stehens, Gehens, während dem Tabak eine ansgesprochene Rolle nicht zugestanden werden kann (Beob. 3). Von nicht $\mathrm{zu}$ unterschätzender Bedeutung ist das Vorkommen von Anomalien an anderen Arterien und an den Radialpulsen, auf die bereits Goldflam und Higier als Zeichen einer angeborenen abnormen Gefässanlage hingewiesen haben und die sich in Beob. 2 und 3 vorfanden. Der Lues kommt ebenfalls eine ätiologische, vielleicht sogar eine aggravierende $\mathrm{Be}$ deutung zu; in einem Falle bestand auch Plattfuss. Das nervös-konstitutionelle Moment seheint nicht von grosser Bedeutung zu sein. Das Vorkommen von Zucker und Eiweiss dürfte wohl bei diesen Kranken häufig auf eine konkurrierende arteriosklerotische Affektion des Pankreas oder der Niere zu beziehen, also als eine Begleiterscheinang und nicht als die Ursache aufzufassen sein. Die "nervöse" Form der D. a. ist mir recht häufig vorgekommen - ich habe es vorgezogen, diese Fälle in meiner Arbeit nicht weiter zu verwerten, weil man bei der Abwesenheit des massgebenden objektiven Befundes - konstantes Fehlen der Fusspulse und bei den unpräzisen Angaben der nervösen Kranken leicht den sicheren Boden der exakten Beobachtung verliert und Gefahr läuft, diese zuerst von Oppenheim beschriebenen Zustände mit Erkrankungen zu verwechseln, die prinzipiell von der D. a. zu trennen sind.

Bezüglich der Literatur wäre noch auf eine gut orientierende und auf persönlichen Erfabrungen basierende Arbeit von G. Muskat hinzuweisen (Sammlung klinischer Vorträge von Volkmann, Chirurgie Nr. 124. 1906). Endlich bitte ich zwei sinnentstellende Druckfehler in meinem Aufsatz korrigieren zu wollen: Seite 278, 7. Zeile von oben - 2 Jahre in Beobachtung, Seite 279, 21. Zeile von unten - krampfartigen Charakter. 